\title{
Improved axle detection for bridge weigh-in-motion systems using fiber optic sensors
}

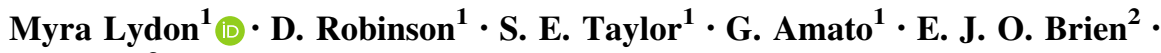 \\ N. Uddin $^{3}$
}

Received: 25 January 2017/Revised: 16 June 2017 / Accepted: 20 June 2017 / Published online: 12 July 2017

(C) The Author(s) 2017. This article is an open access publication

\begin{abstract}
Bridge weigh-in-motion (B-WIM) systems provide a non-destructive means of gathering traffic loading information by using an existing bridge as a weighing scale to determine the weights of vehicles passing over. In this research critical locations for sensors for the next-generation B-WIM were determined from a full 3D explicit finite element analysis (FEA) model. Although fiber optic sensors (FOS) have become increasingly popular in SHM systems there are currently no commercially available fiber optic WIM systems available. The FEA in this research facilitated the development of the first ever full fiber optic B-WIM and its potential has been demonstrated with the site installation of this system. The system combined nothing-on-the-road axle detection and alternative methods of measuring strain at the supports. The system was installed on a 20-m span beam and slab RC bridge in Northern Ireland and the results presented in this paper confirm the suitability of FOS in providing the clear defined peaks required for accurate axle detection in B-WIM.
\end{abstract}

Keywords Bridge weigh-in-motion · Finite element analysis · Fiber optic sensors · Structural health monitoring

Myra Lydon

m.lydon@qub.ac.uk

1 School of Natural and Built Environment, David Keir Building, Queens University Belfast, Belfast BT95AG, UK

2 School of Civil, Structural and Environmental Engineering, University College Dublin, Dublin 4, Ireland

3 Department of Civil, Construction and Environmental Engineering, University of Alabama at Birmingham, Birmingham, USA

\section{Introduction}

The 2013 report card for America's infrastructure states that an annual investment of $\$ 20.5$ billion is required to eliminate the backlog of all cost-beneficial US bridge needs by 2028 [1]. Long-term financial planning is required to maximize the potential of this investment by strategic management of aging infrastructure. B-WIM provides a tool for determining the cause of changes in strain and providing information to facilitate the control of overloaded vehicles [2]. Therefore, it can provide a solution for the long-term monitoring of our infrastructure. The B-WIM theory has been extended under a number of research initiatives [3-6], and more recently the "BridgeMon" project which finished in 2014 [7]. More recently, B-WIM systems have been used in conjunction with machine learning techniques to develop damage detection methods for railway bridges [8]. Previous research [5] has developed theoretical models for B-WIM and demonstrated that Tikhonov Regularization can be used to improve ill-conditioned Moses equations which occur when axles are closely spaced relative to the bridge span. More recently, moving force identification (MFI) techniques have been applied to measured signals to improve the accuracy of the measured axle weights $[6,9,10]$. These techniques have been found to improve the accuracy of the systems [5]. A review of global state-of-the-art of B-WIM systems by the authors [11] was carried out to inform on the development of the next-generation B-WIM with improved accuracy. The findings indicated that improved accuracy could be obtained by optimizing sensor placement, using FEA, by informed selection of scanning frequency and sensor type and critical site selection.

Significant improvements in accuracy of B-WIM systems can be made through the implementation or a shear 
strain based B-WIM as proposed by Bao et al. [12]. In this case a number of field investigations were carried out to assess the suitability of calculating the axle spacing, axle weights and vehicle speed from the shear strain response measured by a strain rosette arrangement rather the traditional method which uses the flexural response of the structure. The distance of the rosette from the support varied in each test case and the findings confirm that the method is less susceptible to dynamic effects due to the sharp peaks in the signal. Further laboratory and field trials were carried out by Kalhori et al. [13] whereby the sensitivity of the location of shear rosette along the bridge span was tested. It was concluded from both laboratory and field trials that the rosette fails to identify individual axles in closely spaced groups if placed at or near mid span. However, it was observed that shear strains collected near the support can reliably increase the number of successful axle identifications, even with groups of closely spaced axles. It was also determined that in cases of multiple presence of vehicles in the same lane the shear strain signal was able to produce discontinuity due to each loading and correctly identify individual vehicles. The existing research indicates that the measurement of shear strain at or near the bridge support can provide a viable method of improving the accuracy of B-WIM systems on a range of bridge types. The applicability of B-WIM systems is currently limited by the type of bridge structure and perceived difficulties in axle detection for stiff or skewed bridges. This research focuses on development and field testing of a B-WIM system for an integral skewed bridge with prestressed beam and in situ slab construction. A 3D explicit FEA model was developed which predicted the suitability of this type of structure for B-WIM applications prior to the site installation. Traditionally B-WIM systems used electric resistance strain gauges; however, there has been extensive research demonstrating the suitability of fiber optic sensors (FOS) for SHM applications [14]. The paper demonstrates the potential of FOS specifically for B-WIM; the FOS B-WIM system was installed on a bridge structure in Northern Ireland and calibration was carried out using only the nothing on the road NOR axle detecting sensors and flexural measurements. A selection of vehicles were also selected from the live flow of traffic at the structure to confirm the presence of clear peaks representing individual axles of vehicles with a gross vehicle weight (GVW) of less than $4 \mathrm{t}$.

\section{Selection of bridge site for B-WIM installation}

The bridge selected is located at Loughbrickland, Co. Down in Northern Ireland and forms an underpass to the main A1 highway connecting Dublin and Belfast, with a static weigh station located lees than a mile north of the site. The integral bridge has a span of $19 \mathrm{~m}$ and an angle of skew of $22.7^{\circ}$; the superstructure consists of 27 Y4 prestressed pre-cast concrete beams which support a $200-\mathrm{mm}$ cast in situ concrete deck slab. The structural detail is shown in Fig. 1. The guidelines for the selection of a suitable B-WIM site are outlined in COST 323 [15] and Table 3 of the specification recommends that a skew of less than $10^{\circ}$ is optimal $[3,16]$. A survey of the bridges on this major route showed that all of them have an angle of skew above $10^{\circ}$. This structure provided the opportunity to meet the challenge of extending the application of B-WIM systems to skew bridges which represent a large portion of bridge stock globally.

Due to the complexity of the structure in comparison to previous B-WIM case studies it was determined that an FEA would provide a valuable insight into the suitability of the structure prior to the site installation.

\section{Explicit finite element analysis (FEA)}

One of the justifications of the developments of the FEA was to confirm that the results required for a successful B-WIM system could be obtained from a structure with pre-stressed members and a skew above $10^{\circ}$. The measurement of shear strains at or near the support has been proven to be successful in other bridge types; in this case the stiffness of the structure raised concerns about its suitability for this new method of axle detection. The numerical model was developed from the as-built drawings using ABAQUS CAE. In order to determine the most effective orientation to measure strain, 8-node linear brick elements with reduced integration (C3D8R) were used to represent the bridge geometry. The concrete had a density of $2.3 \times 10^{3} \mathrm{~kg} / \mathrm{m}^{3}$, elastic modulus of $26.6 \times 10^{9} \mathrm{~N} / \mathrm{m}^{2}$ and Poisson's ratio of 0.2. The steel for the reinforcement and the pre-stress tendons had a density of $8.5 \times 10^{3} \mathrm{~kg} / \mathrm{m}^{3}$, elastic modulus of $205 \times 10^{9} \mathrm{~N} / \mathrm{m}^{2}$ and a Poisson's ratio of 0.3 . The loading for the model represented a typical 5-axle articulated truck with axle spacing's of 3.8, 6.05, 1.25 and $1.3 \mathrm{~m}$ and axle weights of $7.2,14.4,8.8,9.4$ and $8.9 \mathrm{t}$ for axles 1 through 5, respectively. Double tires were assumed for all but the steer axle. The area representing the wheel contact surface of patch loads for the vehicle was taken from BS EN 1991-2 (2003). The axle loads were assumed to be evenly distributed between the wheels, and the wheel loads were distributed uniformly in the rectangular patch. This is a reasonable assumption given the straight road alignment at the bridge. The modeling of the moving-load was treated as a contact analysis by defining a contact interface between the vehicle load part and slab part. An 


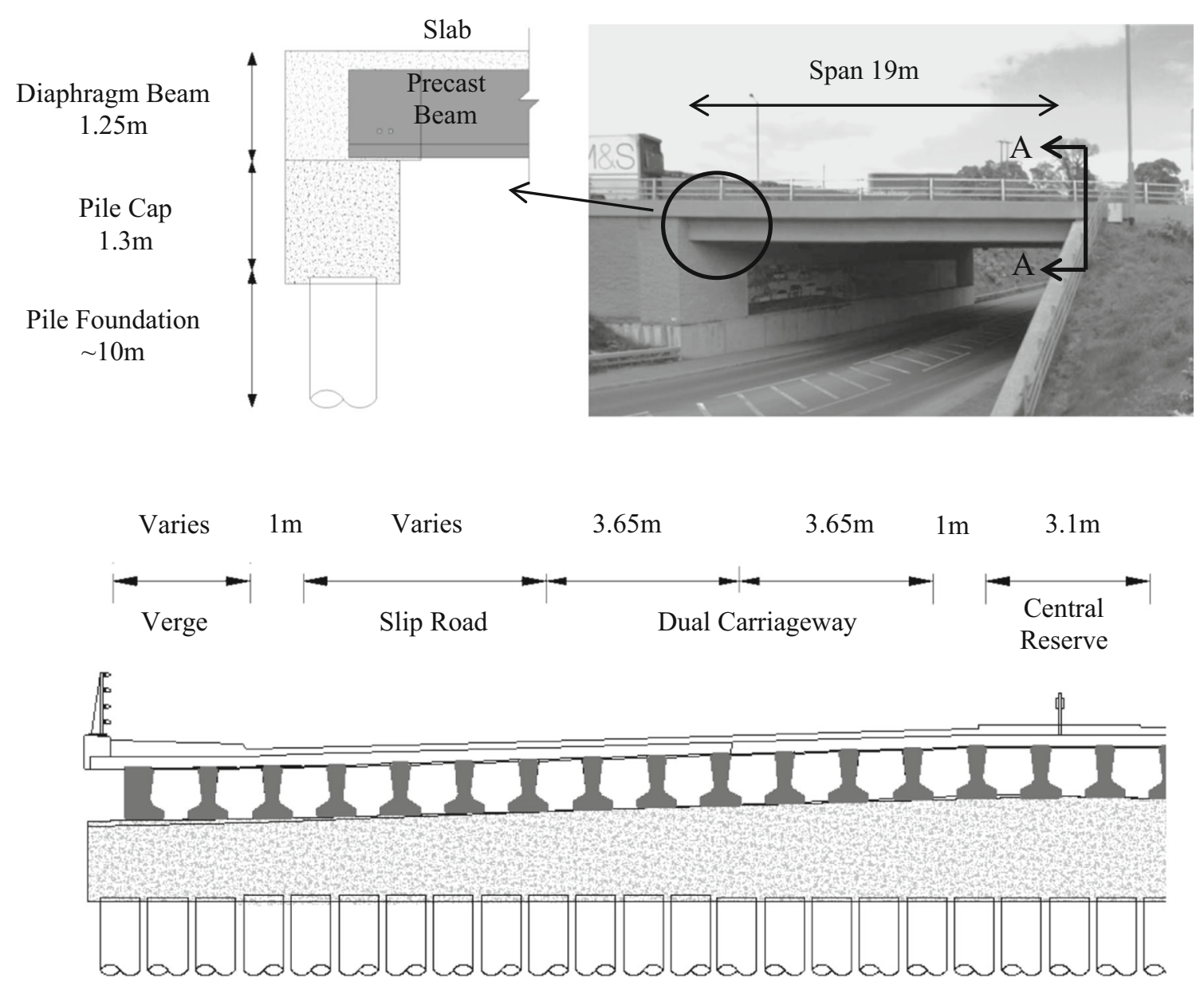

Section A-A: South Abutment Northbound lane

Fig. 1 Bridge structure elevation and section

explicit surface-to-surface interaction was defined between the two parts. A frictionless contact model was adapted which ignored the friction force between the vehicle and the bridge; the authors acknowledge that surface roughness can affect vehicle bounce and dynamic amplitude but in order to simplify the model it was not initially considered.

\subsection{FEA results}

Critically, the FEA indicated that contrary to previous research this type of bridge structure was suitable for B-WIM if a strain amplification method was incorporated [17]. The optimum location for weighing sensors has been well established as the midspan of the longitudinal beams. However, as previously discussed the location of axle detection sensors, and their accuracy, is often the critical factor in improving the accuracy of B-WIM systems.
Therefore, an additional objective of the FEA was to assess the suitability of the locations proposed in previous research $[12,13]$ for this type of structure.

\subsection{Predicting the position of the axle detecting sensors using FEA}

In previous research, NOR axle detecting sensors were attached to the underside of the slab and used to measure a change in transverse strain in the slab as the axle moves over the sensor location [18]. To determine the velocity and axle spacing, two longitudinal sensor locations are required, generally at longitudinal quarter span. The velocity can be calculated by dividing the known distance between the sensors by the time taken by the vehicle to pass between them. The accuracy of this method is largely dependent on the location of the wheel relative to the girders underneath. As shown in 
Fig. 2 Predicted change in transverse strain ( $X$ direction) in slab element a due to moving load over FEA b when wheel load passes over the support girder in FEA
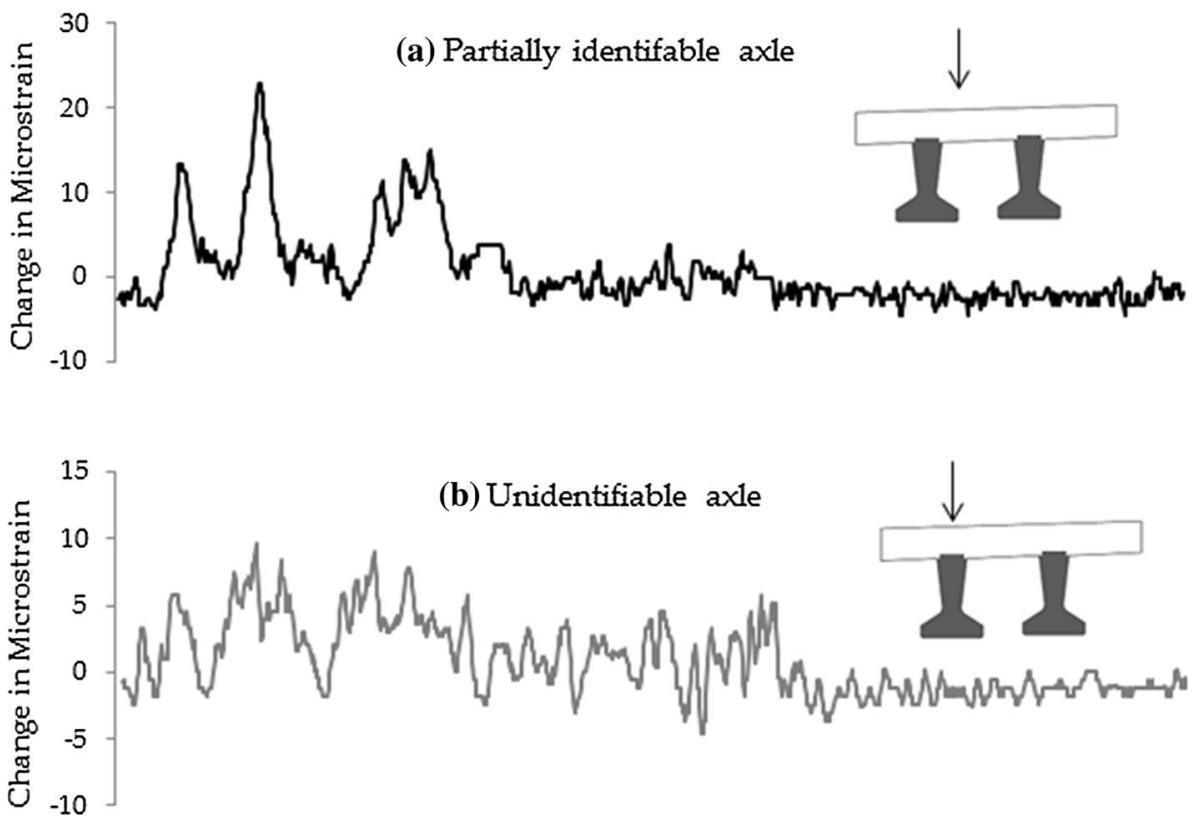

Fig. 2 if the wheel load is applied directly above the girder then the peaks from the individual axle become unidentifiable. Alternative locations were tested in the FEA model for strain sensitivity under the moving load in an attempt to overcome this limiting issue for accuracy in current B-WIM systems.

In line with previous research it was found that the change in strain measured in the vertical direction on the upper side face of the girder near the support region provided clear axle detection. The results (Shown in Fig. 3) indicate that the upper section of the longitudinal girder was subject to compressive strains and this area provided an alternative method for axle detection. During the site installation sensors at this location were fixed labeled new axle detector (NAD). Consistently low strains were predicted in the FEA suggesting a method of strain amplification would be required on site. The details of the amplification achieved and the plate geometry can be found in [19].

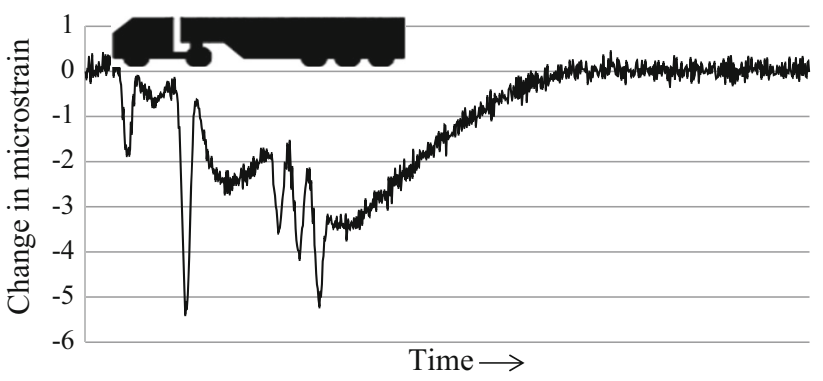

Fig. 3 Predicted change in strain ( $Y$ direction) at NAD location in LR model

\section{Site installation of B-WIM system}

On completion of the FEA and laboratory sensor development [20] the system was installed on site, the final sensor locations are shown in Fig. 4. The installation was carried out in a number of stages in order to minimize disruption to the live carriageway beneath the bridge. A full pavement weigh-in-motion (P-WIM) system was also installed on the approach to the structure to provide validation of the FOS B-WIM system.

\subsection{B-WIM data analysis}

The B-WIM system installed at Loughbrickland can be used for continuous monitoring of the structure and the live traffic. The scanning frequency for the interrogation system was set to $500 \mathrm{~Hz}$. The strain signals collected on site were analyzed in two ways. First a data management software package was used to visualize the signals. This was done to determine if the sensors were sensitive enough to provide the distinct peaks required for axle detection. Second, selections of strain history files for pre-weighed vehicles crossing the structure were selected for calibration using an existing B-WIM software package. Additional information on the software can be found in previous literature [23]. The calibration truck chosen was a 6-axle articulated truck of $30.25 \mathrm{t} \mathrm{GVW}$ as determined at a certified static scale. As the calibration truck was selected from live traffic, only one calibration run was completed. For future testing of this system a better approximation for the influence line could be obtained from an average of at least ten calibration runs. 
Fig. 4 Plan showing locations of sensors installed in lane 1 of northbound carriageway

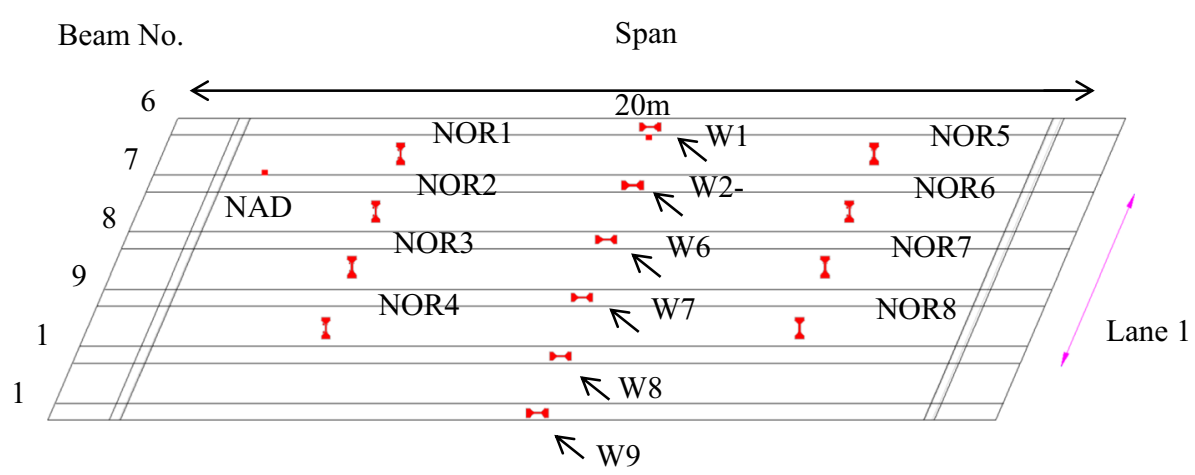

Subsequent vehicles used in the accuracy classification were selected from live traffic and weighed statically at a verified weigh station.

\subsection{NOR axle detection}

One of the goals of this research was to investigate the sensitivity of FOS for axle detection compared to the electrical sensors which are traditionally used in commercial B-WIM systems. The main challenge for NOR is to increase the number of successful axle identifications, particularly when using less suitable structures, such as integral bridges. Current axle detection data generally require significant post processing to identify axles, such as wavelet transforms but the signals are still sensitive to interference from bridge vibrations [21]. The figures provided in this section confirm the suitability of FOS to provide accurate axle detection in a particularly stiff structure. This data were chosen to allow for the comparison in axle detection for a wide range of vehicle types rather than the single vehicle used for the calibration of the P-WIM system.

A moving average filter has been applied to each strain signal presented, as it is common practice to filter the signals. This filtering method was found to be sufficient to remove any false peaks from the signals and allow for clear axle detection. The vehicle types are identified in the graphs using the UK Highways Agency vehicle classification categories. The selection of vehicles has been presented in Fig. 5 and the corresponding weights have been obtained from the P-WIM system.

The results have demonstrated that the next-generation B-WIM system installed on site can provide accurate axle detection data. However, this method of axle detection is largely dependent on the transverse position of the wheel load, as previously discussed. To demonstrate this, the response of one NOR sensor to two different transverse truck locations is presented in Fig. 6. Fig. 6 a demonstrates clear axle detection was obtained for a partially loaded (22 t) Class 52 vehicle. However, Fig. 6b shows an overloaded $(41 \mathrm{t})$ Class 52 vehicle where neither the axles of
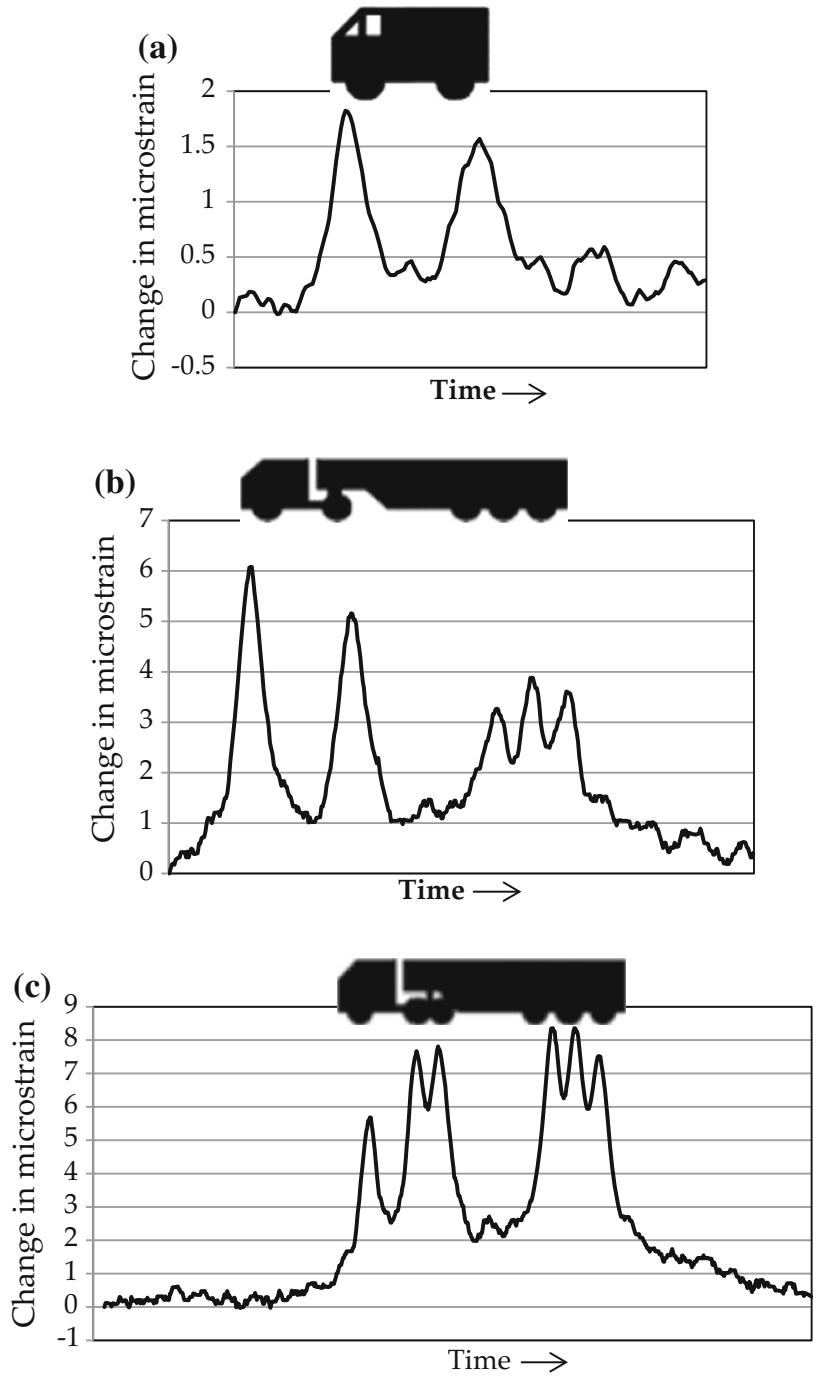

Fig. 5 a Clear peak detection from Class 2 vehicle: 1.78 and $2.07 \mathrm{t}$ respective axle weights. b Clear peak detection from Class 55 unloaded vehicle ( $21 \mathrm{t} \mathrm{GVW})$. c Clear peak detection from Class 56 loaded vehicle (49 t GVW)

the tandem nor the individual axles of the tractor are distinguishable. In order to overcome this issue the alternative strategies were assessed. 

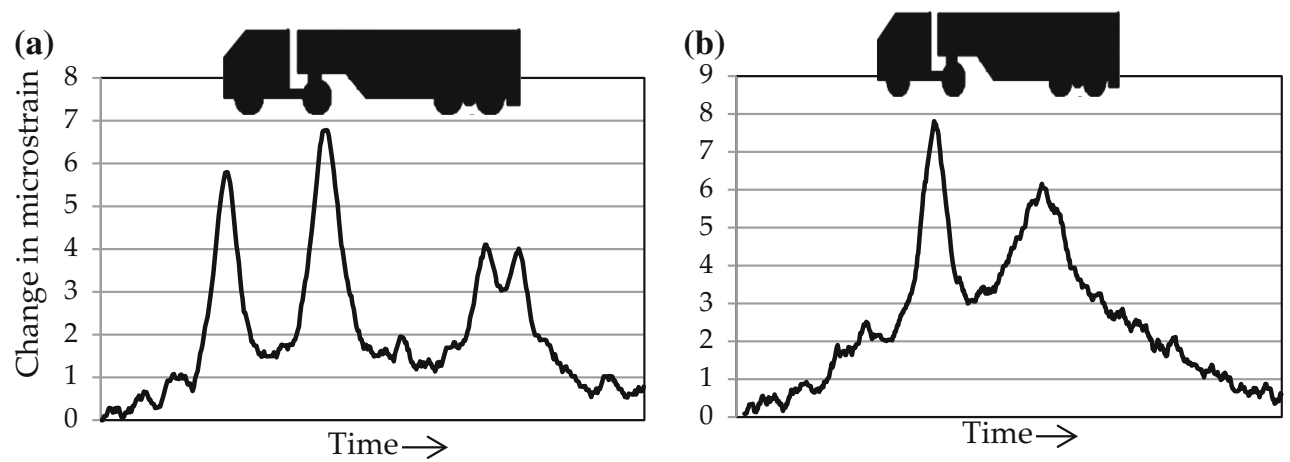

Fig. 6 a Clear peak detection from Class 52 unloaded vehicle-wheel load occurs over slab. b Smeared peak detection from Class 52 loaded vehicle-wheel load occurs over beam

Fig. 7 a NAD sensor positioning b Change in strain due to 5-axle truck measured at this location (a)

$0.8 \mathrm{~m}$ from diaphragm beam (b)

NOR location (at quarterspan)

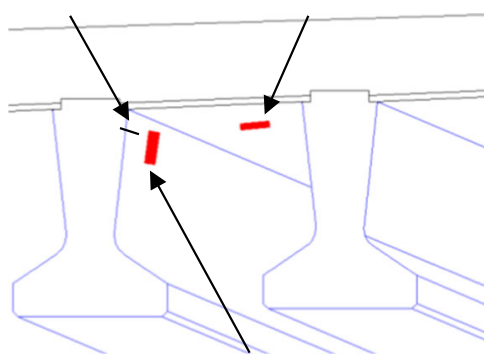

NAD location (near support)

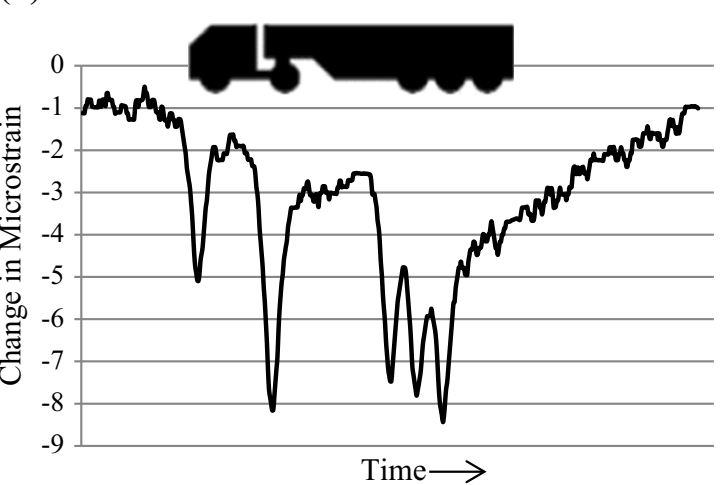

\subsection{New strategies for axle detection}

The measurement of vertical strain on the supporting beams was assessed as an alternative axle detection strategy to instrumenting the slab, shown as NAD in Fig. 7a. An initial trial was carried out to test the new approach. Unfortunately, the system was not time synchronized with the P-WIM system for this trial. Therefore, accurate weights for the presented vehicles were not known. The results from the NAD sensor installed at the support provide well-defined peaks. In Fig. $7 \mathrm{~b}$ a, 5-axle truck can

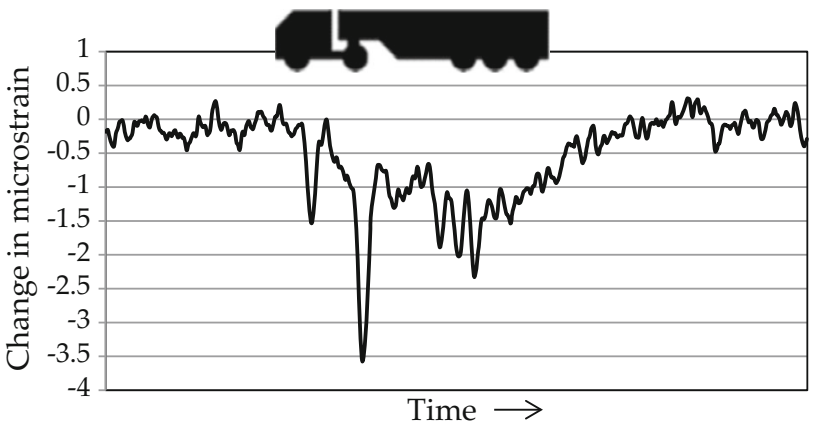

Fig. 8 Peak detection of unloaded Class 55 vehicle using new strategy for axle detection clearly be identified, demonstrating compressive strain measured in the beam can be used as an accurate method of axle detection.

To rigorously test the strategy, clear axle detection must be obtained for an unloaded vehicle. The second axle of the class 55 vehicle presented in Fig. 8 is significantly heavier than the rear tridem. This indicates that the truck was likely to be empty or carrying a light load. Even with a light load the individual peaks can be clearly distinguished for the tridem at the rear. The suitability of this is reinforced in Fig. 9 where all six axles can be

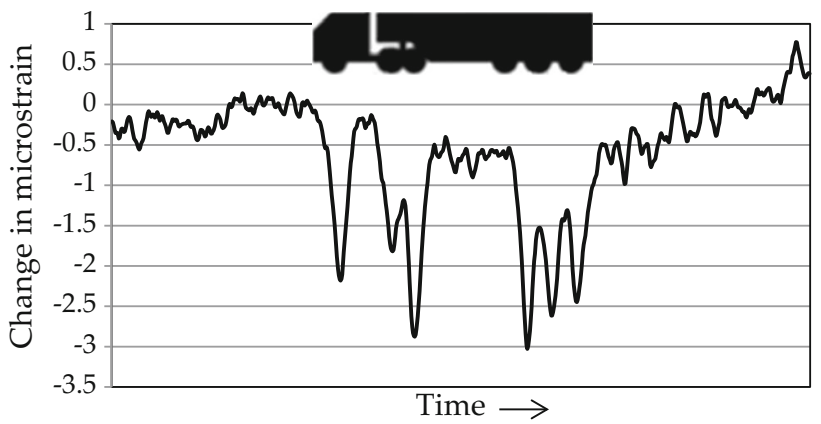

Fig. 9 Peak detection of loaded Class 56 vehicle using new strategy for axle detection 
Table 1 Accuracy classification FOS B-WIM system ( $\delta$ interval width required to satisfy this class; $\pi_{\mathrm{o}}$ minimum required level of confidence; $\pi$ actual level of confidence)

\begin{tabular}{|c|c|c|c|c|c|c|c|c|}
\hline Criterion & Number & Identified (\%) & Mean error $(\%)$ & Standard deviation $(\%)$ & $\delta(\%)$ & $\pi_{\mathrm{o}}(\%)$ & $\pi(\%)$ & Class \\
\hline Gross weight & 6 & 100.0 & -4.50 & 2.45 & 10 & 60.8 & 85.5 & B (10) \\
\hline Group of axles & 8 & 100.0 & -6.14 & 5.79 & 18 & 71.5 & 86.1 & $C(15)$ \\
\hline Single axle & 28 & 97.0 & -2.25 & 9.33 & 20 & 87.1 & 90.1 & $C(15)$ \\
\hline Axle of group & 20 & 95.0 & -2.11 & 9.81 & 20 & 84.9 & 85.9 & B (10) \\
\hline
\end{tabular}

clearly defined even in the case of the closely spaced group to the front of the vehicle. This demonstrates that compressive strain measured in the beam can be used as an accurate method of axle detection.

\subsection{Accuracy of B-WIM system}

The test of the Loughbrickland B-WIM system was carried out under full reproducibility conditions (R2) defined as the test was deemed to be under limited environmental reproducibility conditions (II). The accuracy class determined from the site results are presented in Table 1.

The overall accuracy of the system is $\mathrm{C}(15)$, groups of axles and single axles being the governing criteria. Class B(10) accuracy has been achieved for GVW and axles of a group. The mean errors for all groups are negative which means the system is under weighing. This is possibly caused by some of the static element of the signal being removed during the filtering process. These results can be put in context by considering accuracy classifications obtained from a large-scale test of six WIM systems on an urban roadway in Zürich, Switzerland. Gross weights from some thousands of statically weighed vehicles were used to determine the levels of gross weight accuracy for each system, with reference to COST 323. Of the six systems considered, one was placed in Class $\mathrm{C}(15)$, and two were placed in Class $\mathrm{D}+(20)$. The other three systems were placed in Class E [22]. By comparison this makes the system at Loughbrickland in line with the top accuracy class obtained from established B-WIM sites. Recent sensitivity studies on the accuracy of B-WIM systems have found that a minimum increase of one accuracy class can be achieved by applying individual calibration factors to different types of vehicles [7] and further improvements can be made if:

- The influence line was calculated as the arithmetic mean of a number of influence lines calculated from a number of runs of the calibration vehicle.

- The calibration truck completed a number of runs with slightly varying transverse position.

- A number of calibration factors were determined for varying vehicle types.
- Different influence lines were used for different vehicle speeds.

Future testing of this system will involve more rigorous calibration of the system to allow for this. It is acknowledged that this is a small sample, particularly for gross weights and groups of axles.

\section{Conclusions}

A FEA model has been developed to predict the behavior of a real bridge structure which was chosen as a suitable location for the next-generation B-WIM. The results confirmed that the FEA is a valuable tool and that it provided accurate predictions of the true bridge behavior. The FEA enabled the identification of the critical locations for installation of the first fiber optic B-WIM sensors on the bridge. The FEA also provided an early warning that a method of strain amplification would be required; this led to the development and implementation of a specialized fixing plate.

The FEA model provided accurate predictions of the bridge behavior and allowed for the testing of alternative methods of axle detection. Improved axle detection increases the accuracy of B-WIM systems by increasing the number of correctly identified axle configurations. Successful axle detection is dependent on the transverse position of the vehicle on the bridge; the finding of this research indicates the ideal system is one which combines axle detection sensors at both NOR and NAD locations.

Acknowledgements The authors acknowledge the financial support of DEL, Invest Northern Ireland, Science Foundation Ireland and the United States National Science Foundation for this research. The assistance of the Technical Staff at Queens University Belfast and the staff at Cestel and ZAG (SiWIM) is sincerely appreciated. The authors would also like to thank the Northern Ireland Roads Service and Transport NI for their cooperation throughout this research. Funding was provided by US-Ireland (Grant No. USI023).

Open Access This article is distributed under the terms of the Creative Commons Attribution 4.0 International License (http://crea tivecommons.org/licenses/by/4.0/), which permits unrestricted use, distribution, and reproduction in any medium, provided you give appropriate credit to the original author(s) and the source, provide a 
link to the Creative Commons license, and indicate if changes were made.

\section{References}

1. ACSE (2013) Report card for America's infrastructure. Am Soc Civ Eng 2013:1-74. doi:10.1061/9780784478837

2. Helmi K, Bakht B, Mufti A (2014) Accurate measurements of gross vehicle weight through bridge weigh-in-motion: a case study. J Civ Struct Health Monit 4:195-208. doi:10.1007/s13349014-0076-5

3. Jacob B (2000) Assessment of the accuracy and classification of weigh-in-motion systems. Part 1: statistical background. Int J Veh Des Heavy Veh Syst 7:136-152

4. Jacob B (2002) Weigh-in-motion of axles and vehicles for Europe (WAVE), General Report. Paris

5. Rowley CW, OBrien EJ, Gonzalez A, Žnidarič A (2008) Experimental testing of a moving force identification bridge weigh-in-motion algorithm. Exp Mech 49:743-746. doi:10.1007/ s11340-008-9188-3

6. OBrien E, Rowley CW, González A, Green M (2009) A regularised solution to the bridge weigh in motion equations. Int $\mathrm{J}$ Heavy Veh Syst 16:310-327

7. Corbaly R, Žnidarič A, Leahy C, Kalin J, Hajializadeh D, Zupan E et al (2014) Algorithms for improved accuracy of static bridgeWIM system. D3.1, Report of Bridgemon project, Trzin, Cestal

8. Gonzalez I, Karoumi R (2015) BWIM aided damage detection in bridges using machine learning. J Civ Struct Health Monit 5:715-725. doi:10.1007/s13349-015-0137-4

9. Dowling J, OBrien EJ, González A (2012) Adaptation of cross entropy optimisation to a dynamic bridge WIM calibration problem. Eng Struct 44:13-22. doi:10.1016/j.engstruct.2012.05. 047

10. Law SS, Fang Y (2001) Moving force identification: optimal state estimation approach. J Sound Vib 239:233-254

11. Lydon M, Taylor S, Mufti A, OBrien E (2016) Recnet developments in bridge weigh in motion. J Civ Struct Health Monit 6(1):69-81

12. Bao T, Babanajad SK, Taylor T, Ansari F (2016) Generalized method and monitoring technique for shear-strain-based bridge weigh-in-motion. J Bridge Eng 21:1-13. doi:10.1061/(ASCE)BE. 1943-5592.0000782

13. Kalhori H, Alamdari MM, Zhu X, Samali B, Musttapha S (2017) Non-intrusive schemes for speed and axle identification in bridgeweigh-in-motion systems. Meas Sci Technol. doi:10.1088/1361$6501 / \mathrm{aa} 52 \mathrm{ec}$

14. Glisic DHSB (2015) On-site validation of fiber-optic methods for structural health monitoring: Streicker bridge. J Civ Struct Health Monit 5:529-549. doi:10.1007/s13349-015-0123-x

15. COST323 (1999) Weigh-in-motion of road vehicles: final report. Paris

16. Jacob B, O'Brien E, Newton W (2000) Assessment of the accuracy and classification of weigh-in-motion systems. Part 2: European specification. J Veh Des Heavy Veh Syst 7:153-168

17. Schmidt F, Jacob B (2012) Experimentation of a bridge WIM system in France and applications to bridge monitoring and overload screening. 6th International Conference Weigh-In-Motion (ICWIM 6), Dallas, pp 33-42

18. Kalin J, Žnidarič A, Lavrič I (2006) Practical implementation of nothing-on-the-road bridge weigh-in-motion system. International symposium on heavy vehicle weights and dimensions

19. Lydon M, Taylor S, Robinson D (2014) Development of a bridge weigh-in-motion sensor: performancecomparison using fibre optic and electric resistance strain sensor systems. IEEE Sens J 14:4284-4296

20. Lydon M, Taylor S, Robinson D, Doherty C, Callender P (2013) Assessment of various sensors for structural health monitoring for bridge weigh-in-motion (B-WIM). 6th International Conference Structure Health Monitoring Intelligent Infrastructure, Hong Kong

21. Kim S, Lee J, Park M, Jo B (2009) Vehicle signal analysis using artificial neural networks for a bridge weigh-in-motion system. Sensors 9:7943-7956. doi:10.3390/s91007943

22. O'Connor A, Brien EJO (2005) Traffic load modelling and factors influencing the accuracy of predicted extremes. Can J Civ Eng 32:270-278. doi:10.1139/L04-092

23. Žnidarič A, Lavrič I, Kalin J, Kulauzović B (2011) SiWIM Bridge Weigh-in-Motion Manual, 4th edn. Cestel in Trzin, Slovenia 